\title{
O PLANEJAMENTO ESTRATÉGICO E A VISÃO BASEADA EM RECURSOS (RBV) \\ uma avaliação da tecnologia da informação na gestão hospitalar
}

\section{1- Saulo Barbara de Oliveira*}

Doutor em Engenharia de Produção pela Universidade Federal do Rio de Janeiro (UFRJ/COPPE), Brasil.

Professor Adjunto no Programa de Pós-Graduação em Administração na Universidade Federal Rural do Rio de Janeiro (UFRRJ), Brasil.

saulobarbara@gmail.com

http://lattes.cnpq.br/0247628533800844

\section{2- Favio Akiyoshi Toda}

Doutorando em Administração pela Pontifícia Universidade Católica do Rio de Janeiro (PUC/Rio), Brasil.

Professor Assistente no Programa de Graduação em Administração da Universidade Federal Rural do Rio de Janeiro (UFRRJ), Brasil

favio.toda@uol.com.br

http://lattes.cnpq.br/206413551174972 


\section{O PLANEJAMENTO ESTRATÉGICO E A VISÃO BASEADA EM RECURSOS (RBV): UMA AVALIAÇÃO DA TECNOLOGIA DA INFORMAÇÃO NA GESTÃO HOSPITALAR}

\section{RESUMO}

O artigo apresenta os resultados de uma pesquisa empírica conduzida em cinco hospitais públicos da cidade do Rio de Janeiro. Traz em destaque o caso de um hospital público que poderá servir de benchmarking para outros hospitais brasileiros, uma vez que tem na Tecnologia da Informação (TI) e no Planejamento Estratégico (PE) um valioso ativo para o desenvolvimento de suas competências, indo ao encontro da teoria RBV (resource-based view). A pesquisa, de natureza qualitativa e exploratória analisa a percepção dos gestores de hospitais sobre a situação em que se encontram a consciência, as ações e as práticas da gestão estratégica de $\mathrm{TI}$. Os dados de campo foram coletados em entrevistas feitas com os gestores de $\mathrm{Tl}$ e de infraestrutura desses hospitais. O estudo possibilitou identificar carências e problemas relativos ao PE, seleção, implantação e uso da TI, e de outras ferramentas nos hospitais investigados. Os resultados são preocupantes e apresentam relatos do quanto a gestão hospitalar precisa ser aprimorada, especialmente no tocante ao reconhecimento da $\mathrm{TI}$ como recurso valioso para o desenvolvimento de competências organizacionais e inovações.

\section{Palavras-chave}

Gestão Hospitalar, Visão Baseada em Recursos, Planejamento Estratégico, Tecnologia da informação.

\section{THE STRATEGIC PLAN AND THE RESOURCE-BASED VIEW (RBV): AN EVALUATION OF INFORMATION TECHNOLOGY IN HOSPITAL MANAGEMENT}

\section{ABSTRACT}

This article presents the results of empirical research conducted in five public hospitals of Rio de Janeiro city. It Highlights the case of a public hospital that can serve as a benchmarking for other brazilian hospitals since it has in information technology (IT) and in strategic planning (SP) a valuable asset for the development of their skills, going to meet the RBV theory (resource-based view). This qualitative and exploratory study examines the hospitals managers' perception on the situation of strategic awareness, organizational practices and actions to IT strategic management. The field data were collected in interviews with IT and infrastructure managers of these hospitals. The study made it possible to identify shortcomings and problems relating to the SP, selection, deployment and use of IT and others tools in hospitals researched. The results are worrying and present reports of how much the hospital management needs to be improved, especially as regards the recognition of IT as a valuable resource for developing organizational skills and innovations.

\section{Keywords}

Hospital Management; Resource-based View; Strategic Planning; Information Technology. 


\section{Introdução}

Os hospitais são espaços de atuação que requerem um diálogo salutar de diferentes lógicas de conhecimento e entendimento entre os profissionais de saúde, administrativos e pacientes, em busca da harmonia e de resultados positivos (Zarama-Vasquez \& Vinck, 2008). Daí a importância do desenvolvimento de um plano coordenado e sistematizado para a melhoria do desempenho das instituições de saúde. Independente de ter ou não fins lucrativos, a existência de qualquer instituição está centrada em sua capacidade de captar e manter clientes por meio de produtos e serviços diferenciados que atendam as suas necessidades (Levitt, 1985).

A TI tem função relevante para o desenvolvimento de inovações e o aprimoramento dos serviços hospitalares, tornando-se necessária a gestão profissionalizada em diferentes frentes de trabalho (gerência de rede, base de dados, segurança, etc.), em grande parte devido ao uso de softwares especializados, os quais vão desde o controle de registro de pacientes, passando pelo diagnóstico e até a mais recente aplicação: a tele medicina (Otto, Catanho, Degrave \& Miranda, 2007; Capretz, Toledo, Fantinato, Garcia, Wang, Allison, Nabuco, Rodrigues, Bonacin, Sasse, Gimenes \& Cunha, 2009).

Assim, o conhecimento de TI e a habilidade em gestão tornaram-se competências indispensáveis para os gestores lidarem com as atividades de planejamento, execução e controle hospitalar (Capretz et al., 2009). Isso se torna cada vez mais importante na medida em as organizações de qualquer tipo e tamanho se esforçam para se conectarem e usar os recursos da Internet, especialmente serviços online ou Web Services (Aghaei, Nematbakhsh \& Farsani, 2012).

Já no início do século XX. Henry Fayol destacava que entre as funções empresariais básicas (Fabricação/Produção, Comercial, Financeira, Segurança, Contábil e Administração) a Administração era a mais negligenciada de todas. Nela estariam inseridas atividades comuns a qualquer tipo de organização: planejamento, organização, comando, coordenação e controle (Stoner \& Freeman, 1985). Decorrido quase um século, é ainda de senso comum, que o planejamento continua sendo negligenciado em boa parte das organizações. Entretanto, a dedicação de estudiosos sobre o tema continua se ampliando significativamente. Estudos pioneiros na área de planejamento estratégico (PE) começaram com Ken Andrews, Ansoff, Alfred Chandler e se estendem atualmente para Porter, Mintzberg, passando também pelas ideias de Drucker (Prahalad \& Hamel, 2005).

Considerando o moderno campo da Administração Estratégica, a Visão Baseada em Recursos, ou o seu acrônimo RBV (resource-based view), ganhou ampla aceitação acadêmica e corporativa por considerar, que em essência, a fonte de vantagem competitiva está nos recursos e não em função de como a empresa se posiciona em relação ao ambiente externo (Barney, 1996).

Em razão da importância socioeconômica que os hospitais públicos detêm perante a sociedade, a recorrência de problemas divulgados pelos veículos de comunicação sobre a gestão hospitalar e a insatisfação da população quanto aos serviços prestados, o presente trabalho se preocupa em avaliar a gestão de TI em hospitais públicos na cidade do Rio de Janeiro, importante área para o aprimoramento da eficiência organizacional e um recurso valioso para o desenvolvimento das competências hospitalares na prestação de serviços à sociedade.

A TI pode ser vista como um recurso estratégico que possibilita aos hospitais a desenvolverem suas competências tecnológicas ampliando seus portfólios de serviços. Por exemplo, o Hospital Sírio-Libanês (HSL) foi o primeiro na América Latina a ter um Centro de Desenvolvimento e Treinamento em cirurgia robótica, e o primeiro no Brasil a utilizar um robô em uma cirurgia. Em 2003, incorporou também serviços de tele medicina, interligando em sistema de videoconferência, os treinamentos em vídeo cirurgia, micro cirurgia, artroscopia e braquiterapia da próstata (Editora Finep, 2011).

Neste trabalho propõe-se a analisar como a Tecnologia da Informação é administrada em hospitais públicos da cidade do Rio de Janeiro, por meio da seguinte questão de pesquisa: "Qual a situação em que se encontram a consciência, as ações e as práticas da gestão de TI nos hospitais do Rio de Janeiro sob uma perspectiva estratégica?"2.

\section{O Planejamento Estratégico}

Antes de 1970, os hospitais norte-americanos eram essencialmente da esfera pública, sem fins lucrativos e recorriam pouco ao PE. A maior preocupação era com o resultado financeiro entre os custos e o 
reembolso. De 1970 a 1980, o PE consistia basicamente em desenvolver novas infraestruturas para aumentar o orçamento oriundo do Governo, que regulava o setor. A entrada de empresas privadas no setor, fez com que o PE tivesse maior importância em razão do acirramento da competição e da percepção de incerteza dos gestores no mercado, onde a estratégia principal era a maximização do lucro (Zukerman, 2005). Esta descrição da dinâmica do segmento hospitalar apresentado para os Estados Unidos pode ser vista também como um bom retrato para o contexto brasileiro.

De 2000 em diante, o PE apresenta uma abordagem diferenciada observando aspectos contingenciais que afetam os negócios. Para Zukerman (2005), a construção do plano estratégico consiste de quatro atividades: (a) revisão organizacional incluindo a missão, filosofia e a cultura organizacional; (b) avaliação do ambiente externo, da estrutura do mercado e sua dinâmica; (c) avaliação das características internas distintas da organização; (d) análise da posição competitiva da instituição, observando vantagens e desvantagens.

Embora exista uma variedade de formas com que o PE é realizado nas organizações de saúde, seja qual for a opção escolhida, seus líderes julgam que o PE é uma prática importante e necessária (Mintzberg, Ahlstrand \& Lampel,1998). Várias ferramentas colaboram na formulação das estratégias e sua implementação, entre as quais se destacam a matriz SWOT (Strenghs, Weakness, Opportunities, and Threats) e o BSC (Balanced Scorecard).

O modelo SWOT é empregado na avaliação das variáveis internas (forças e fraquezas) e externas (ameaças e oportunidades) das organizações (Mintzberg et al., 1998). Embora seja um modelo tradicional, continua sendo uma ferramenta central no campo da Administração para formulação de estratégias. O Balanced Scorecard de Kaplan e Norton (1992) é outra ferramenta de gestão (Borba, 2011) e sua grande aceitação nas empresas deve-se à sua capacidade de articulação entre as ações a serem executadas e os objetivos, que estão vinculados por medidas de avaliação e relacionados às seguintes perspectivas: (1) financeira, (2) clientes, (3) processos internos, e (4) inovação e aprendizagem.

Entretanto, o cumprimento desses objetivos requer que as empresas estejam capacitadas, motivo pelo qual o desenvolvimento das competências organizacionais transformou-se em importante tema na literatura da Administração Estratégica (Barney, 1996; Mintzberg et al., 1998; Doz \& Hamel, 1998; Eisenhardt \& Martin, 2000; Prahalad \& Hamel, 2005; Newbert, 2007; Tellis, Prahbu \& Chand, 2009), fortalecendo uma linha teórica dominante conhecida como RBV (resource-based view), uma teoria mais próxima da realidade da Administração Pública, pela inexistência de concorrentes diretos. Tellis et al. (2009) observam a preocupação de empresas inovadoras com a construção de uma cultura forte e uma relativa despreocupação com a concorrência na formulação das estratégias, alertando para a importância das competências organizacionais.

A teoria da RBV teve início com a obra de Penrose em 1959 que propunha uma nova maneira de interpretar o papel da empresa como uma organização estruturada em um conjunto de recursos. Com os trabalhos de Wernerfelt (1984), Barney (1991) e Peteraf (1993), a partir da década de 80, a teoria teve impulso baseada nas considerações desses autores de que os recursos internos da organização constituem fonte de vantagem competitiva e que as diferenças individuais entre as empresas devem ser consideradas na formulação da sua estratégia.

Para Barney e Hesterly (2007), os recursos são definidos como ativos tangíveis (fábrica, por exemplo) e intangíveis (reputação entre os clientes) que a empresa controla e que estariam disponíveis na elaboração e execução das estratégias. As capacidades são vistas como um subconjunto dos recursos da empresa que permitiriam utilizar outros recursos que ela possui a disposição.

Em uma orientação diferenciada sobre as competências ou capacitações, Schreyögg e Kliesch-Ebertl (2007) destacam que não se trata de um recurso único (capital, tecnologia, recursos humanos), mas sim uma forma distinta e superior de alocar recursos que surge de interações sociais e representam uma forma coletiva de "resolução de problemas". As competências são resultados de processos de aprendizado organizacional e não de um plano corporativo. Os autores observam que as competências só existem quando um conjunto de habilidades comprovadamente resolveu problemas complexos; referem-se a padrões rotineiros e replicáveis de resolução de problemas, devendo funcionar de forma confiável (ser bem sucedida ao longo do tempo em diferentes situações) e ser reproduzível.

Doz e Hamel (1998) lembram que as empresas precisam identificar suas competências e preencher os gaps existentes, recomendando que para preenchê-los, os gestores façam alianças estratégicas. Artmann e Rivera (2003) citam esta importante dimensão da gestão hospitalar que trata das parcerias 
necessárias para o desenvolvimento das competências organizacionais ao propor estratégias colaborativas entre hospitais, com base na teoria desenvolvida por Crémadez e Grateau, a qual defende a responsabilidade do hospital na articulação de redes com outras instituições.

As parcerias, especialmente aquelas exercidas por meio de redes de colaboração entre empresas, são fundamentais para o fortalecimento de intercâmbios, troca de informações, conhecimento e experiências. E as empresas hoje estão cientes dos benefícios potenciais relacionados com a sua adesão em redes de colaboração empresarial (Cheikhrouhou, Pouly, \& Choudhary, 2011). Sguario e Tomaél (2011, p. 58) corroboram com esse pensamento ao afirmarem que "o desenvolvimento de um sistema capaz de disseminar e compartilhar facilmente as informações corporativas torna-se essencial para a integração das ações e o intercâmbio de ideias".

Eisenhardt e Martin (2000) também contribuem para a teoria RBV inserindo uma dimensão temporal importante sobre a capacidade de adaptação às mudanças do ambiente, ou seja, as estratégias vistas como atividades de capacitação e trabalhadas de forma dinâmica frente às questões de oportunidades e ameaças ao longo do tempo (Dynamic Capabilities). Para Teece (2007), a capacidade dinâmica é uma meta-competência que transcende a competência operacional e que pode ser conceituada a partir da perspectiva RBV, embora enfatize que esta teoria tenha um viés estático. O autor considera que se a empresa possui recursos ou competências estáticas, pode mantém-se competitiva por um curto espaço de tempo, mas não irá se sustentar no longo prazo, a menos que tenha a sorte ao seu lado. As empresas com capacidade dinâmica moldam a competição e o mercado, o que Ihes possibilitam ser mais empreendedoras e inovadoras.

Para Shengbin (2011), muitas empresas dão grande importância à Pesquisa e Desenvolvimento (P\&D) empregando pessoal altamente qualificado, criando uma boa cultura e contratando mais recursos tecnológicos. No entanto, essas atividades nem sempre estão direcionadas a atender a demanda do mercado ou para o desenvolvimento organizacional, o que faz com que o desempenho da inovação nem sempre seja alto.

Entretanto, a inovação hospitalar, seja por inovações médicas (novos tratamentos ou métodos de diagnóstico relacionados à atividade fim do hospital) ou por inovações administrativas (relativo à estrutura organizacional e processos administrativos) é vista por Wu e Hsieh (2011) como um recurso valioso e único para manter a competitividade de acordo com a teoria RBV. Estes autores observam que as inovações administrativas impactam mais a percepção dos clientes sobre a qualidade da assistência do que as inovações médicas (Wu \& Hsieh, 2011).

Bryson, Ackermann e Eden (2007) destacam que uma chave para o sucesso de organizações públicas é a identificação e a construção de capacitações estratégicas na produção de valor para os stakeholders a um custo razoável. Seguindo nesta linha de pensamento os autores salientam que esta capacitação deve possuir também uma característica dinâmica, dado que é necessário para o alcance deste objetivo um desejável ajuste com o ambiente que é mutável ao longo do tempo. É oportuno observar que esses autores descrevem a aplicação de uma metodologia, baseada na perspectiva RBV, que apóia a elaboração do plano estratégico na Beeches Management Centre (órgão ligado ao Sistema de Saúde na Irlanda do Norte em fornecer suporte em gestão para outras organizações), onde se busca identificar e desenvolver as competências para alcançar os objetivos desta instituição (Bryson et al., 2007).

A construção de planos estratégicos consistentes interna e externamente, isto é, adequados aos recursos disponíveis, com razoável grau de risco, projetados para um horizonte de tempo adequado e funcional a nível corporativo e departamental, e à luz da RBV, configura-se uma competência essencial de uma organização. Isso contribui para ampliar a aprendizagem coletiva que busca conciliar os diversos saberes específicos (acumulação e aplicação de conhecimentos em produção, vendas, logística, estratégia, recursos humanos, etc.) para o alcance dos objetivos empresariais. A percepção positiva que os executivos têm sobre a elaboração do planejamento estratégico, sua realização, e sobre o desenvolvimento de parcerias, representa um importante recurso intangível organizacional no aperfeiçoamento de sua competência. Teece (2007), de certo modo, corrobora com este pensamento ao afirmar que as capacidades dinâmicas estão fortemente relacionadas à equipe gerencial, e que estas causam impactos nos processos organizacionais, sistemas e na estrutura existentes nas organizações. 


\section{A Gestão Estratégica da TI nos hospitais}

Os hospitais são organizações complexas por possuírem uma extensa e variada cadeia de valor e relacionamentos com diferentes entidades, que envolve um grande número de processos administrativos, produtivos financeiros, tecnológicos, de negócios, de práticas de saúde (diagnósticos e tratamentos de enfermidades) e hoteleiros (hospedagem, alimentação e repouso).

Outro ponto para a reflexão estratégica é o fato de que os hospitais apresentam custos altos, em razão da natureza e características dos seus suprimentos, os quais são intensivos em tecnologia e em conhecimento. Ao tratar de vidas humanas, sua gestão requer atenção, cuidados especiais, visão e ação estratégicas na definição de prioridades dos investimentos em tecnologia e na sua utilização, onde uma simples falha pode comprometer o seu futuro.

O uso crescente de novas e sofisticadas tecnologias nos últimos vinte anos produziram em todos os setores da economia uma complexidade organizacional sem precedente que resultou em novos desafios para as instituições ao ampliar a capacidade de registro, armazenamento, análise, controle e transmissão em tempo real de grandes volumes de informações (Obrien \& Maracas, 2007). A TI tem transformado as empresas em suas formas de trabalho, no modo como se decidem e até a estrutura e cultura organizacional.

A racionalização dos processos de trabalho tem sido objeto de atenção nas empresas que buscam melhorar o fluxo de informação e atividades, reduzir o tempo do ciclo de produção, maximizar o uso de insumos, espaços físicos, procedimentos e recursos humanos, aumentar a produtividade e qualidade, maximizar o lucro e a competitividade das empresas. A própria indústria de cuidados da saúde "está cada vez mais se tornando uma comunidade baseada no conhecimento, ligando hospitais, clínicas e os pacientes para o compartilhamento de conhecimento, reduzindo os custos administrativos e melhorando a qualidade do atendimento" (Pinochet, 2007; Barbosa, Gonçalves \& Simonetti, 2009, p.45).

Entretanto, os sistemas eletrônicos de registro de pacientes estão entre os que mais precisam de melhorias. Como afirmam Côrtes e Côrtes (2011) a baixa qualidade da informação disponível, os problemas de acesso e o armazenamento de um grande número de registros dificultam a investigação científica e o desenvolvimento de análises retrospectivas na medicina.

Desse modo, os sistemas informatizados devem ser cada vez mais usados pelos hospitais com objetivo de melhorar o tratamento e a integração das informações da área médica, buscando a automatização dos processos de trabalho em saúde.

Porém, tradicionalmente nas organizações de saúde, a não adoção da TI é motivada na resistência dos seus profissionais, embora essa postura esteja mudando (Spil \& Schuring, 2006). Aparecem também na literatura relatos negativos de casos sobre dificuldades enfrentadas pelos funcionários de organizações de saúde, devido a pouca familiaridade com a TI (Albuquerque, Prado \& Machado, 2011). Além disso, "as próprias instituições de ensino de saúde enfrentam o desafio de aumentar a produção de conhecimento sobre o uso de tecnologias de informação em pesquisa e na gestão do sistema de saúde" (Cavalcante \& Vasconcellos, 2007, p.23).

Portanto, não é de se estranhar que a $\mathrm{TI}$ e os Sistemas de Informação (SI) tornaram-se fatores críticos de sucesso por proporcionarem recursos e facilidades para se lidar com a complexidade da gestão hospitalar (Resende, 2003). Contudo, a sua aplicação tem sido lenta, parcial e gradual porque os serviços hospitalares apresentam características peculiares, tanto em sua estrutura, quanto na gestão estratégica e operacional. Isso requer modelos de gestão mais completos e com fluxo de informação e processos decisórios específicos (Freitas \& Rech, 2003).

Por outro lado, a interação das pessoas com a informática também exige atenção, uma vez que a introdução de novas tecnologias leva em conta o modo como os diferentes usuários lidarão com elas, o que determina as consequências da sua adoção (Almeida \& Melllo, 2004; Queiroz \& Moreira, 2007; Albuquerque, 2009).

Quanto a sua governança a TI oferece desafios próprios como "a necessidade de uma melhor compreensão e visibilidade de seus processos internos, o total alinhamento com os objetivos de negócios e a operação econômica de sua infraestrutura" (Duque \& Lyra 2010, p.39).

Escrivão (2007, p.9), numa pesquisa sobre a tomada de decisão e sistemas de informação em 24 hospitais públicos estaduais da administração direta da Região Metropolitana de São Paulo, acrescenta que 
"os hospitais produzem um considerável volume de dados, embora com importantes lacunas, e que muitos gestores desconhecem a existência de tais informações ou não as utilizam adequadamente para subsidiar a gestão hospitalar".

\section{Metodologia}

Utilizando-se da taxionomia de Vergara (2000), esta pesquisa pode ser classificada quanto aos seus fins como exploratória (na medida em que busca compreender mais sobre a forma como a Tl é gerenciada atualmente dentro dos hospitais), e descritiva (ao expor sobre as características de gestão e práticas).

Quanto aos meios de investigação, trata-se de uma pesquisa empírica, qualitativa, realizada em cinco hospitais públicos localizados na cidade do Rio de Janeiro, na qual vinte hospitais, escolhidos pela proximidade da região central da cidade do Rio de Janeiro foram convidados a participar da pesquisa. O convite foi feito por carta dirigida ao principal executivo destes hospitais. Em cada um dos hospitais participantes, dois dirigentes, o responsável pela gestão da infraestrutura e outro da gestão de TI foram os respondentes da pesquisa.

As entrevistas foram agendadas diretamente com os sujeitos da pesquisa, com antecedência em torno de 20 dias, e realizadas no local de trabalho dos entrevistados. No momento do agendamento os sujeitos eram orientados a reservar com exclusividade toda a parte de uma manhã para as entrevistas, devendo ainda ler as questões e se preparar para as entrevistas com base num roteiro semiestruturado (Apêndice I), enviado por e-mail logo após o agendamento.

Por se tratar de uma pesquisa de natureza qualitativa, visando buscar a validade e confiabilidade dos resultados, optou-se por seguir a orientação de Paiva Júnior, Sousa Leão e Mello (2011), por meio da descrição clara, rica e detalhada, tanto da pesquisa quanto da análise dos resultados.

Para Martins (2006) a confiabilidade é capacidade de um instrumento de pesquisa (entrevistas, questionários, etc.) de satisfazer as exigências necessárias para a finalidade de uso, nos limites préestabelecidos, os quais foram especificados para manter suas propriedades por um período determinado. Para isso, foi feito um teste piloto do roteiro de entrevista com quatro sujeitos potenciais da pesquisa.

Por outro lado, quanto ao critério surpresa citado por Paiva Júnior et al. (2011), embora não se tenha chegado à saturação dos dados com base nas entrevistas nos cinco hospitais, observou-se na pesquisa uma variedade de formas de manejo da $\mathrm{TI}$ como recurso estratégico, o que corrobora em parte com a percepção de outros profissionais de TI da área da Saúde que dialogaram com os pesquisadores em vários momentos desta pesquisa. Porém, uma grata surpresa ocorreu em verificar um exemplo, em uma das instituições, a utilização adequada da TI como um recurso para a obtenção de vantagem competitiva.

As entrevistas foram conduzidas pelo principal autor deste artigo, que realizou integralmente o levantamento destes dados primários. Foram feitas entre julho e agosto de 2010, durando cada uma cerca de duas horas, começando com o executivo da infraestrutura e prosseguindo com o executivo de TI.

De posse de um notebook e o roteiro como guia, as perguntas eram formuladas e suas respostas imediatamente registradas pelo entrevistador abaixo das respectivas questões no roteiro, em arquivo digital, o que dispensou a necessidade de transcrição dos dados.

O tratamento dos dados foi realizado por meio da análise de conteúdo, de acordo com o que propõe Vergara (2010, p.7), que reconhece ser esta uma técnica apropriada para identificar "o que está sendo dito sobre um determinado tema", e colabora na análise de categorias, que segundo Bardin (2009), é a técnica mais antiga e ainda uma das mais utilizadas para o desdobramento do texto em unidades e o seu reagrupamento em categorias analógicas que identificam o que estas têm em comum, a partir do que emergem do texto.

Com base nestas técnicas foi feita a análise qualitativa dos dados, organizados em duas categorias, a primeira sobre a consciência estratégica e ações dos hospitais, e a segunda quanto às práticas organizacionais relacionadas ao desenvolvimento de competências da gestão estratégica de TI, envolvendo atividades de Pesquisa e Desenvolvimento (P\&D) e formação de parcerias.

Com a proposta de avaliar a área de Tecnologia da Informação dos hospitais sob a perspectiva da teoria RBV, considerou-se que para ser vista como um recurso estratégico pelos hospitais no desenvolvimento de suas competências organizacionais seria necessário o levantamento das atividades de 
planejamento realizadas e quais eram as iniciativas que existiam para o aprimoramento destas competências.

A pesquisa realizada não permite a generalização dos resultados para a população de hospitais da cidade do Rio de Janeiro, pelo fato de ser uma amostra pequena e não aleatória, porém admitem-se a possibilidade de que outros hospitais públicos dessa cidade, do estado e do país possam ser identificados com algum dos casos estudados, e que as recomendações feitas no presente estudo possam ser aplicáveis a esses e outros hospitais.

\section{Análise e discussão dos resultados}

Por questão de confidencialidade os hospitais avaliados foram identificados por letras do alfabeto. Os hospitais A, B e C estão vinculados a universidades públicas, o D é vinculado diretamente ao Governo Federal e o hospital E está ligado a Secretaria de Saúde do Estado do Rio de Janeiro. Os hospitais B e D são considerados referências em suas especialidades no país e ganharam notoriedade nos meios de comunicação nos últimos anos por tratarem de enfermidades que têm grande repercussão na sociedade.

\section{Hospital A}

Como visto antes, este é um hospital vinculado a uma universidade pública. Contava com cerca de 730 funcionários e 600 leitos para atendimento. Apresentou o número de 55.000 atendimentos por ano com internações e 750 atendimentos laboratoriais anualmente.

\section{Consciência estratégica e ação}

O hospital realiza a atividade de planejamento estratégico de modo sistemático com periodicidade de revisão entre seis meses a um ano. O PE envolve a alta direção e a média gerência, e ocorre o desdobramento deste plano estratégico em Planos de Ação. Os projetos estratégicos são de conhecimento parcial dos funcionários do nível operacional e, neste caso, poucos são os funcionários que participam do PE. Para o acompanhamento e avaliação das estratégias usa somente o ciclo PDCA (Plan-Do-CheckAct/Planejar-Fazer-Checar-Agir).

Os estudos, dos quais as estratégias de negócio são desenvolvidas estão baseados apenas na análise da demanda atual e potencial. Tal comportamento reflete a necessidade de que o PE passe a ser realizado de forma a envolver e integrar representantes das atividades essências do hospital.

O hospital não possui certificado de acreditação, e também não adota um sistema de qualidade baseado nas normas ISO (normas internacionais de padronização) ou equivalente, como também não utiliza um método de controle de qualidade como o 5 S (Seiri - utilização/descarte, Seiton - ordenação, Seisou - higiene, Seiketsu - padronização e Shitsuke - disciplina), Programa de Idéias e Sugestões, ou qualquer outro.

A direção do hospital acredita no uso intensivo da TI como recurso importante para a competitividade organizacional. Porém, o hospital não apresenta mecanismos de monitoramento do ambiente externo, tais como o surgimento de novas tecnologias, estratégias dos concorrentes e interesses dos clientes.

Nos últimos três anos o investimento em inovação tecnológica ficou entre $2 \%$ a $3 \%$ do orçamento. Para o ano seguinte, estava previsto aplicar o mesmo percentual, caso o orçamento da instituição se mantivesse nos níveis dos anos anteriores. Na percepção dos seus gestores, o principal entrave à inovação tecnológica não é o investimentos em tecnologia, mas a baixa qualificação de seu pessoal.

\section{Tl, Pesquisa \& Desenvolvimento e Parcerias}

Na avaliação das atividades de $P \& D$, os executivos atribuíram grau de importância média. Sobre as atividades de aquisição de conhecimentos externos, os gestores atribuíram o mesmo grau de importância, com a observação de que esta atividade é realizada de forma ocasional.

Entre os investimentos em TI nas áreas de Administração, Operações, Sistemas de Almoxarifado, ERP (Sistema de Gestão), CRM (Sistema de Relacionamento com Clientes), EAD (Sistema de Ensino a Distância), tele medicina e outros que poderiam ser especificados pelo respondente, o hospital A prevê investir apenas em sistemas de gestão integrada do tipo ERP. Ao mesmo tempo, registra-se a existência de esforços para a 
qualificação dos seus profissionais para os trabalhos em $\mathrm{Tl}$ e Internet. As principais prioridades da instituição em relação à inovação tecnológica estão voltadas para automatizar a gestão e melhorar a informatização do hospital.

O Hospital declara possuir parceria com uma entidade pública para o desenvolvimento de inovação tecnológica. Conhece os tipos de financiamento existentes e linhas de crédito ou incentivo governamental para a inovação tecnológica, onde já foi utilizado este recurso para o projeto de rede de comunicação de dados.

Na busca da avaliação do nível de importância sobre as entidades que interagem com o hospital, verificam-se a seguir a percepção dessa importância e os tipos de cooperação realizados com o hospital, mostrados na Figura 1.

\begin{tabular}{|c|c|c|}
\hline Relações entre o hospital e: & Importância & Tipo de Cooperação \\
\hline Clientes & Média & $P \& D$ \\
\hline Fornecedores & Não relevante & Assistência Técnica \\
\hline Outro hospital & Baixa & Assistência Técnica \\
\hline Empresas de consultoria & Não relevante & Ensaios para testes de produtos \\
\hline Universidades e institutos de pesquisa & Média & Assistência Técnica \\
\hline $\begin{array}{l}\text { Centros de capacitação profissional e } \\
\text { assistência técnica }\end{array}$ & Média & Treinamento \\
\hline
\end{tabular}

Figura 1: Importância e tipo de cooperação entre o hospital e outras entidades

Observa-se não haver a prática de parcerias para o desenvolvimento das suas competências científicas (voltadas para as atividades fins do hospital) e tecnológicas (voltadas para as atividades meio dos hospitais, nas quais se encontra a TI). Isso reflete, no entendimento dos pesquisadores, a existência de uma visão estratégica estreita e deficiência no desenvolvimento de competências por parte da instituição.

\section{Hospital B}

Também vinculado a uma universidade pública, este hospital contava com quase 700 funcionários e próximo de 200 leitos. Realiza cerca de 3.600 atendimentos com internações e pouco mais de 135 mil atendimentos ambulatoriais, por ano.

\section{Consciência estratégica e ação}

Este hospital não realiza PE, e suas estratégias são formuladas com base na intuição dos seus dirigentes e de maneira informal. A instituição não possui acreditação, ou qualquer outro tipo de certificação, e não utiliza programa voltado à qualidade dos serviços hospitalares.

Apesar de vir investindo mais de $4 \%$ do seu orçamento anualmente, e prosseguir neste patamar, o hospital B parece ser o que mais apresenta dificuldades em desenvolver a inovação tecnológica. Além da baixa qualificação do pessoal (o único entre os cinco hospitais que avalia o seu corpo de profissionais como não suficientemente capaz para a implantação da TI), registra também problemas com a verba disponível para investimentos em razão da sua dependência de dotação orçamentária, colocada como principal entrave para investimentos em $\mathrm{Tl}$.

\section{TI, Pesquisa \& Desenvolvimento e Parcerias}

Apesar dos problemas de ordem financeira, a instituição reconhece a importância da inovação tecnológica e desenvolveu arranjos cooperativos com outras organizações com vistas a promover atividades inovadoras, principalmente com fornecedores e com outros hospitais. Porém, seus executivos desconhecem linhas de financiamento, de crédito ou incentivos governamentais para a inovação tecnológica. A preocupação em termos de aplicação da inovação tecnológica está na área de operações, na automatização da gestão e no uso de base de dados para armazenar informações de pacientes. $80 \%$ dos investimentos em $P \& D$ deste hospital são feitos sempre com grandes empresas estrangeiras. Isso provavelmente ocorre devido às características da enfermidade na qual este hospital é especialista, cujo know-how se concentra nessas empresas fornecedoras.

A Figura 2 apresenta o nível de importância e os tipos de cooperação encontrados na relação do hospital com outras entidades, onde é possível perceber que o relacionamento com clientes e fornecedores é altamente importante para este hospital. 


\begin{tabular}{|l|c|c|}
\hline \multicolumn{1}{|c|}{ Relações entre o hospital e: } & Importância & Tipo de Cooperação \\
\hline Clientes & Alta & P\&D \\
\hline Fornecedores & Alta & $\begin{array}{c}\text { P\&D, Assistência Técnica, Treinamento, } \\
\text { Ensaios para testes de produtos, Outras } \\
\text { atividades de cooperação }\end{array}$ \\
\hline Outro hospital & Média & $\begin{array}{c}\text { P\&D, Assistência Técnica, Treinamento, } \\
\text { Ensaios para testes de produtos, Outras } \\
\text { atividades de cooperação }\end{array}$ \\
\hline Empresas de consultoria & $\begin{array}{c}\text { Não se aplica } \\
\text { Universidades e institutos de pesquisa }\end{array}$ & Não relevante \\
\hline $\begin{array}{l}\text { Centros de capacitação profissional e } \\
\text { assistência técnica }\end{array}$ & Não relevante & Não se aplica \\
\hline
\end{tabular}

Figura 2: Importância e tipo de cooperação entre o hospital e outras entidades

Além da importância dada aos clientes e fornecedores quanto ao tipo de cooperação percebida pela instituição, observa-se que os objetivos da cooperação buscam desenvolver competências científicas, porém os pesquisadores observam que as competências tecnológicas parecem não estar sendo levadas em consideração por este hospital.

\section{Hospital C}

O C é outro hospital universitário que tem mais de mil funcionários, 540 leitos, 67 mil atendimentos com internação e 832 mil atendimentos em laboratório, por ano.

\section{Consciência estratégica e ação}

Este hospital, à semelhança do B, não desenvolve PE de forma estruturada e sistematizada, e suas estratégias são elaboradas de modo informal e apoiadas na intuição dos seus dirigentes. Embora estejam vinculados a instâncias superiores distintas, os hospitais B e C apresentam muitas semelhanças. O hospital C, a exemplo do B, não possui acreditação e nem programa voltado à qualidade da prestação dos serviços de saúde.

Apesar de ter uma participação orçamentária destinada à inovação tecnológica um pouco menor (entre $2 \%$ e $3 \%$ nos últimos três anos) do que o hospital B, o C possui um quadro de percepção mais favorável quanto ao fato de considerar a capacidade do seus técnicos suficiente para a implantação de projetos de Tl, e de se preocupar com a qualificação dos funcionários com este objetivo.

\section{Tl, Pesquisa \& Desenvolvimento e Parcerias}

A instituição desconhece linhas de financiamento, de crédito ou incentivos governamentais para a inovação tecnológica. Sua preocupação em termos de aplicação da inovação tecnológica orienta-se para a informatização, automação da gestão e emprego de base de dados para armazenar informações de pacientes. As áreas que prevêem maiores investimentos pensam em destiná-los a atividades mais básicas: gestão administrativa, operações, sistemas de almoxarifado, ERP e uma mais avançada, que seria a tele medicina.

Apesar de perceber como sendo de média importância as inovações que se desenvolveram ao longo dos últimos quatro anos, mesmo assim o hospital $\mathrm{C}$ realizou arranjos cooperativos com outras organizações visando a desenvolver atividades em inovação tecnológica.

A Figura 3 mostra a percepção dos gestores sobre o nível de importância e os tipos de cooperação encontrados na relação do hospital C com outros grupos de entidades, destacando a importância que este hospital atribui ao seu relacionamento com fornecedores, instituições de pesquisa e outros hospitais.

\begin{tabular}{|l|c|c|}
\hline \multicolumn{1}{|c|}{ Relações entre o hospital e: } & Importância & Tipo de Cooperação \\
\hline Clientes & Média & Pesquisa de satisfação \\
\hline Fornecedores & Alta & P\&D \\
\hline Outro hospital & Alta & P\&D \\
\hline Empresas de consultoria & Não relevante & Outras atividades de cooperação \\
\hline Universidades e institutos de pesquisa & Alta & P\&D \\
\hline $\begin{array}{l}\text { Centros de capacitação profissional e } \\
\text { assistência técnica }\end{array}$ & Baixa & Treinamento \\
\hline
\end{tabular}

Figura 3: Importância e tipo de cooperação entre o hospital e outras entidades 
Observa-se também que a instituição é orientada a desenvolver competências científicas, porém os dados levantados não são suficientes para se afirmar se existe ou não o interesse no desenvolvimento de competências tecnológicas.

\section{Hospital D}

O hospital D é vinculado ao Governo Federal e considerado referência em sua área de especialidade com grande projeção na região em que atua. São pouco mais de 800 funcionários, quase 400 leitos, 643.000 atendimentos ambulatoriais e 35.000 internações, por ano.

\section{Consciência estratégica e ação}

Entre os cinco hospitais estudados, o Hospital D é o que se destaca por ser o mais bem estruturado e informatizado, onde os funcionários, independentes dos níveis hierárquicos, participam e se envolvem no PE da instituição. As fontes a partir das quais as estratégias de negócio são desenvolvidas são mais completas do que no hospital A, pois além da análise sobre a demanda atual e potencial de clientes, o D considera também outros levantamentos, tais como a análise de cenários, análise da concorrência, avaliação das ameaças e oportunidades, pesquisa sobre grau de satisfação de clientes, realização de benchmarking, rediscussão sobre a missão e competências reconhecidas.

Enquanto o hospital A emprega para o acompanhamento e reformulação das estratégias o modelo PDCA, o hospital D faz uso de um sistema de gestão integrado próprio. Outra diferença que parece significativa entre ambos, refere-se ao fato de que o hospital D recorre ao uso de uma importante metodologia de avaliação de desempenho organizacional conhecida como indicadores chave de desempenho (Key Performance Indicators - KP), enquanto que o hospital A ainda evidencia a necessidade de buscar um sistema de indicadores capaz de avaliar o seu desempenho.

Observa-se também que dentre as cinco instituições avaliadas, a D é a única a possuir um sistema de gestão da qualidade implantado e a respectiva acreditação desse sistema, desde 2005.

\section{Tl, Pesquisa \& Desenvolvimento e Parcerias}

O hospital D também conhece linhas de financiamento, de crédito ou incentivos governamentais para a inovação tecnológica e já recorreu a linhas vinculadas ao Ministério da Saúde para investir nesta área. O hospital investiu nos últimos três anos mais de $4 \%$ dos seus recursos financeiros e esperava manter este mesmo nível para o ano seguinte. $60 \%$ dos investimentos em inovação foram realizados com grandes fornecedores estrangeiros, semelhante ao que ocorreu com o hospital B e, provavelmente, pelo mesmo motivo: concentração nestas empresas de know-how na área em que este hospital é especialista.

Com relação ao investimento em inovação tecnológica o hospital D é o mais ambicioso de todos e pretende investir em várias áreas. Estão também previstos investimentos na gestão hospitalar, operações, no ensino a distância (EAD) e em um sistema específico, conhecido como Picture Architecture System, um sistema computadorizado de desenho e apresentação de imagens de objetos em movimento com duas ou três dimensões. Na medicina auxilia na realização de diagnósticos por imagem de forma mais rápida e eficiente.

\begin{tabular}{|l|c|c|}
\hline \multicolumn{1}{|c|}{ Relações entre o hospital e: } & Importância & Tipo de Cooperação \\
\hline Clientes & Alta & Pesquisa de satisfação \\
\hline Fornecedores & Alta & $\begin{array}{c}\text { P\&D, Assistência Técnica, Treinamento, Outras } \\
\text { atividades de cooperação }\end{array}$ \\
\hline Outro hospital & Média & $\begin{array}{c}\text { P\&D, Assistência Técnica, Treinamento, Ensaios } \\
\text { para testes de produtos, Outras atividades de } \\
\text { cooperação }\end{array}$ \\
\hline Empresas de consultoria & Baixa & Ensaios para testes de produtos \\
\hline $\begin{array}{l}\text { Universidades e institutos de } \\
\text { pesquisa }\end{array}$ & Média & Trento \\
\hline $\begin{array}{l}\text { Centros de capacitação profissional e } \\
\text { assistência técnica }\end{array}$ & Baixa & \multicolumn{2}{|c|}{\begin{tabular}{c} 
Ensaios para testes de produtos \\
\hline
\end{tabular}}
\end{tabular}

Figura 4: Importância e tipo de cooperação entre o hospital e outras entidades

A instituição destaca que o principal entrave à realização da atividade inovadora é a burocracia. A importância da inovação nos três últimos anos para esta instituição é tida como alta, e entre 2006 e 2010 o 
hospital realizou arranjos cooperativos para desenvolvimento de ações inovativas sem, no entanto, indicar quais foram essas ações.

A Figura 4 descreve a percepção dos gestores sobre a importância e os tipos de cooperação encontrados na relação do hospital D com outras entidades, destacando a alta importância atribuída ao seu relacionamento com clientes e fornecedores.

Semelhante ao que ocorre no hospital C, o D parece também focar o desenvolvimento em competências científicas. Mas, diferente do C, é possível perceber o interesse do hospital D no desenvolvimento de competências tecnológicas.

\section{Hospital E}

O hospital E é vinculado a Secretaria de Saúde do Estado do Rio de Janeiro. Possui quase 450 funcionários e pouco mais de 40 leitos. São realizados em média quase 490.000 atendimentos ambulatoriais e cerca de 400 atendimentos com internação, por ano.

\section{Consciência estratégica e ação}

Apesar de não realizar o planejamento estratégico de modo sistemático, o hospital E declara conduzir o levantamento do ambiente externo em relação à satisfação dos pacientes e busca identificar novas tecnologias. É outro hospital que também não possui acreditação, nem tão pouco um programa de qualidade para a melhoria dos seus serviços. Os pesquisadores destacam como ponto importante as informações que são obtidas sobre a satisfação dos funcionários. Porém, dado ao fato dessas informações não serem incluídas nos planos estratégicos ou programa de qualidade, é possível questionar a eficácia deste levantamento.

\section{TI, Pesquisa \& Desenvolvimento e Parcerias}

Nos últimos três anos o hospital E investiu entre 1\% e 2\% dos seus recursos, por ano, e espera manter este mesmo nível para os anos seguintes. O hospital pretendia para o próximo ano investir na área de inovação tecnológica em sistema de gestão e no controle de estoques (sistema de almoxarifado). A instituição também considera que a principal barreira para a realização da atividade inovativa é a burocracia.

Tal como os hospitais A e D, o E declara ter conhecimento sobre linhas de financiamento, de crédito ou incentivos governamentais para a inovação, sendo que o hospital E, a exemplo do que fez o D, buscou recursos em linhas vinculadas ao Ministério da Saúde. Nos três últimos anos a instituição considerou alta a importância da inovação e desenvolveu, entre 2006 e 2010, arranjos cooperativos voltados para essa finalidade. Cerca de $80 \%$ dos investimentos em P\&D foram realizados com fornecedores de grandes empresas estrangeiras, tal como fizeram os hospitais B e D.

A Figura 5 indica a percepção dos gestores sobre o nível de importância e os tipos de cooperação encontrados na relação do hospital E com outras instituições, destacando a alta importância que este hospital atribui ao seu relacionamento com clientes e fornecedores.

\begin{tabular}{|l|c|c|}
\hline \multicolumn{1}{|c|}{ Relações entre o hospital e: } & Importância & Tipo de Cooperação \\
\hline Clientes & Alta & Pesquisas de satisfação \\
\hline Fornecedores & Alta & $\begin{array}{c}\text { P\&D, Assistência Técnica, Treinamento, } \\
\text { Outras atividades de cooperação }\end{array}$ \\
\hline Outro hospital & Média & $\begin{array}{c}\text { P\&D, Assistência Técnica, Treinamento, } \\
\text { Ensaios para testes de produtos, Outras } \\
\text { atividades de cooperação }\end{array}$ \\
\hline Empresas de consultoria & Ensaios para testes de produtos \\
\hline $\begin{array}{l}\text { Universidades e institutos de pesquisa } \\
\text { assistência técnica }\end{array}$ & Média & Treinamento \\
\hline
\end{tabular}

Figura 5: Importância e tipo de cooperação entre o hospital e outras entidades

O hospital E apresenta um comportamento organizacional semelhante aos dois anteriores quanto aos objetivos e percepção da importância das parceiras. Sua orientação indica estar mais voltada para o desenvolvimento de competências científicas, do que no uso de recursos para o aprimoramento de competências tecnológicas. 
O planejamento estratégico e a visão baseada em recursos (RBV): uma avaliação da tecnologia da informação na gestão hospitalar

\section{Síntese dos resultados}

Visando apresentar uma sintetize dos resultados da pesquisa sobre as categorias analisadas foram elaborados as Figuras 6 e 7.

\begin{tabular}{|c|c|}
\hline \multicolumn{2}{|r|}{ PRINCIPAIS RESULTADOS DA CATEGORIA CONSCIÊNCIA ESTRATÉGICA E AÇÃO } \\
\hline $\begin{array}{l}\bar{\pi} \\
\frac{\pi}{0} \\
\text { 음 } \\
\text { 오 }\end{array}$ & Síntese dos resultados \\
\hline$A$ & $\begin{array}{l}\text { - } \quad \text { Conta com PE sistematizado com avaliação e acompanhamento usando o PDCA, e ainda: a) revê o PE num período } \\
\text { entre seis meses a um ano; b) envolve a alta direção e a média gerência, mas com pouca participação do nível } \\
\text { operacional; c) gera planos de ação e projetos estratégicos } \\
\text { - } \quad \text { Formula suas estratégias somente com base na análise da demanda } \\
\text { - } \quad \text { Não possui acreditação e nem sistema de gestão da qualidade (SGQ) } \\
\text { - } \quad \text { Não conta com mecanismos de avaliação de novas tecnologias, estratégias dos concorrentes e satisfação dos clientes } \\
\text { - Investiu em inovação nos últimos três anos de } 2 \% \text { a } 3 \% \text { do orçamento } \\
\text { - Seu principal entrave à inovação tecnologia: baixa qualificação de pessoal. }\end{array}$ \\
\hline B & $\begin{array}{l}\text { - } \quad \text { Não conta com o PE formalmente instituído } \\
\text { - } \quad \text { Formula suas estratégias somente com base na intuição dos dirigentes } \\
\text { - } \quad \text { Inve possui acreditação e nem SGQ } \\
\text { - } \quad \text { Conta com inovação nos últimos três anos } 4 \% \text { do orçamento } \\
\text { - } \quad \text { Principais entraves à inovação tecnologia: baixa qualificação de seu pessoal e escassez recurso financeiro. }\end{array}$ \\
\hline $\mathrm{C}$ & $\begin{array}{l}\text { - Não conta com o PE formalmente instituído } \\
\text { - } \quad \text { Formula suas estratégias somente com base na intuição dos dirigentes } \\
\text { - } \quad \text { año possui acreditação e nem SGQ } \\
\text { - } \quad \text { Investiu em inovação nos últimos três anos } 3 \% \text { do orçamento } \\
\text { - } \quad \text { Desenvolve com um quadro de pessoal capacitado a implementação de TI }\end{array}$ \\
\hline $\mathrm{D}$ & $\begin{array}{l}\text { - PE bem sistematizado - sua avaliação e acompanhamento são feitos usando sistema próprio e envolve a participação } \\
\text { - } \quad \text { Formula suas estratégias com base na demanda de clientes, análise de cenários, ameaças e oportunidades } \\
\text { - } \quad \text { Avalia a satisfação dos clientes } \\
\text { - Faz benchmarking } \\
\text { - } \quad \text { Faz a avaliação de desempenho organizacional usando modelo KPI } \\
\text { - Possui acreditação e SGQ } \\
\text { - Investiu em inovação nos últimos três anos mais de } 4 \% \text { do orçamento } \\
\text { - Principal entrave à inovação tecnologia: burocracia. }\end{array}$ \\
\hline$E$ & $\begin{array}{l}\text { - Não conta com o PE formalmente instituído } \\
\text { - } \quad \text { Não possui acreditação e nem SGQ } \\
\text { - } \quad \text { Avalia a satisfação dos clientes } \\
\text { - Investiu em inovação nos últimos três anos de } 1 \% \text { a } 2 \% \text { do orçamento } \\
\text { - } \quad \text { Principal entrave à inovação tecnologia: burocracia. }\end{array}$ \\
\hline
\end{tabular}

Figura 6: Consciência estratégica e ação

\begin{tabular}{|c|c|}
\hline \multicolumn{2}{|r|}{ PRINCIPAIS RESULTADOS DA CATEGORIA TI, PESQUISA \& DESENVOLVIMENTO E PARCERIAS } \\
\hline 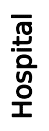 & Síntese dos resultados \\
\hline A & $\begin{array}{l}\text { - } \quad \text { Atribui média importância atribuída às atividades de P\&D e de aquisição de conhecimentos externos } \\
\text { - } \quad \text { Prevê investir em TI em ERP e na qualificação dos profissionais para a Internet } \\
\text { - } \quad \text { Prioriza a inovação tecnológica em gestão e automação } \\
\text { - Tem parceria pública para a inovação tecnológica } \\
\text { - } \quad \text { Conhece linhas de crédito/incentivo governamental e fez uso uma vez para expansão da rede de dados. }\end{array}$ \\
\hline B & $\begin{array}{l}\text { - Reconhece a importância da inovação tecnológica } \\
\text { - } \quad \text { Prioriza a inovação tecnológica nas áreas de operaçães, automatização, gestão e base de dados } \\
\text { - } \quad \text { Desenvolve arranjos cooperativos visando à promoção de atividades inovadoras } \\
\text { - } \quad \text { Desconhece linhas de crédito/incentivo governamental para a inovação tecnológica } \\
\text { - } \quad \text { Atribui grande importância ao relacionamento com clientes e fornecedores. }\end{array}$ \\
\hline
\end{tabular}


Continuação...

\begin{tabular}{|c|c|}
\hline C & $\begin{array}{l}\text { - } \text { Atribui média importância à inovação } \\
\text { - Prioriza a inovação tecnológica nas áreas da gestão administrativa, operacional, almoxarifado e tele medicina } \\
\text { - } \quad \text { Desenvolve arranjos cooperativos para promover atividades inovadoras } \\
\text { - } \quad \text { Desconhece linhas de crédito/incentivo para a inovação tecnológica. }\end{array}$ \\
\hline D & $\begin{array}{l}\text { - } \quad \text { Encontra-se bem informatizado e estruturado } \\
\text { - } \quad \text { A maioria dos seus fornecedores }(60 \%) \text { é de grandes empresas estrangeiras. } \\
\text { - } \quad \text { Prioriza a inovação tecnológica em várias áreas, destacando o ensino a distância e um sistema especialista - Picture } \\
\text { - } \quad \text { Deschitecture System } \\
\text { - } \quad \text { Atribui grande importância ao relacionamento com clientes e fornecedores e outras instituições. }\end{array}$ \\
\hline$E$ & $\begin{array}{l}\text { - Reconhece a importância da inovação e busca identificar novas tecnologias } \\
\text { - } \quad \text { Conhece e usa linhas de crédito/incentivo governamental para investir em inovação tecnológica } \\
\text { - } \quad \text { Prioriza a inovação tecnológica nas áreas da gestão e controle de estoque } \\
\text { - } \quad \text { Desenvolve arranjos cooperativos visando promover atividades inovadoras } \\
\text { - } \quad \text { Atribui grande importância ao relacionamento com clientes e fornecedores. }\end{array}$ \\
\hline
\end{tabular}

Figura 7: Tl, Pesquisa \& Desenvolvimento e Parcerias

\section{Conclusões e Recomendações}

Este estudo identificou problemas e carências relativas à atividade de planejamento nas instituições pesquisadas, possibilitando a constatação de negligência não apenas com a gestão estratégica hospitalar, como também na atividade de desenvolvimento de competências organizacionais por meio da Tecnologia da Informação, recurso estratégico atualmente essencial para a prestação de serviços inovadores para a sociedade. A conclusão é a de que os hospitais avaliados empregam a TI como uma ferramenta importante e necessária, mas a explorada apenas numa perspectiva funcional, pelo fato de ser ainda pouco presente o seu alinhamento aos objetivos estratégicos e às necessidades do negócio.

Felizmente, entre as entrevistas nos cinco hospitais públicos, uma delas destaca-se ao avaliar a TI não apenas como uma atividade de apoio na gestão hospitalar para a função básica de automação, mas como um valioso recurso para o desenvolvimento de suas competências organizacionais e, consequentemente, da sua competitividade que pode ser vista como a capacidade de oferta de soluções efetivas para a sociedade (lembrando que este hospital é uma referência nacional entre hospitais públicos e também privados).

Os autores chamam a atenção para o fato de que as experiências positivas observadas no hospital D poderiam ser bastante úteis e armazenadas para a formação de uma memória organizacional, um repositório de conhecimento, visando o seu compartilhamento entre os parceiros. Esse, sem dúvida, desponta como um caso de referência para a prática de benchmarking entre instituições congêneres, indo ao encontro da teoria RBV que recomenda que as empresas desenvolvam seus recursos (neste caso de TI) visando apoiar a criação de estratégias eficazes.

Para os hospitais que precisam superar as dificuldades e melhorar o PE, poderia ser uma boa opção insistir nas parcerias com outras instituições do mesmo setor com a formação de um grupo multidisciplinar de PE e gestão estratégica de TI com a participação de outros hospitais, do próprio Ministério da Saúde, ou do Ministério do Planejamento, Orçamento e Gestão. Isso não invalida avaliar a possibilidade de contratar consultorias especializadas nesta área, ou desenvolver as competências internamente buscando uma qualificação na área da Administração. Avalia-se também se a criação de fóruns de discussão, tão presentes nas comunidades virtuais, não seria um bom instrumento para fomentar o intercâmbio de aprendizagem, experiências e práticas entre estas instituições e alimentar a memória de gestão organizacional.

Como observado no decorrer das pesquisas no processo de coleta de dados, um dos problemas apontados pelos respondentes como entrave à inovação tecnológica refere-se à falta de mão-de-obra qualificada. Uma vez que a administração hospitalar, conforme salientado no referencial teórico é uma atividade complexa, principalmente em função de conciliar os diversos saberes e competências dos profissionais que atuam no setor de saúde, os pesquisadores colocam as hipóteses de falta de conhecimentos modernos e habilidades na gestão estratégica dos hospitalares pesquisados. Seria também lógico questionar se a resistência dos profissionais de saúde, bem como a dificuldade em usar os recursos de TI, também de acordo com o referencial teórico, não seriam os maiores entraves para a sua adoção nos hospitais pesquisados. 
Quatro dos cinco hospitais estudados, a direção considera suficiente a qualificação do seu pessoal para empreender a implantação da TI quando, ao mesmo tempo, afirma que o principal entrave a inovação tecnológica, em três destes hospitais, seria a qualificação da mão de obra. Na avaliação dos autores isto, de certa forma, evidencia uma contradição quando se observa também uma baixa eficiência destes hospitais no uso de TI.

Em todos os hospitais pesquisados avalia-se que ainda há muito a ser feito na área da TI de maneira a contribuir para um melhor desempenho estratégico e operacional destes hospitais. Deduz-se disso, a necessidade de empreender esforços e recursos voltados para o desenvolvimento de competências tecnológicas, com a esperança de tornar estas instituições mais habilitadas a selecionar, contratar, instalar e manter as ferramentas de TI, mais adequadas a cada hospital.

Quanto a tele medicina, considerando a sua alta potencialidade, observa-se a existência de enorme lacuna no uso desta tecnologia, inclusive no único hospital que a utiliza, pois a utiliza parcialmente. Embora ainda considerada uma inovação nas instituições pesquisadas, e por isso mais difícil de ser implantada, impõe-se a estas instituições o desafio de melhor conhecê-la e usá-la em benefício dos seus pacientes e da sociedade.

Ressalte-se a hipótese de que a consciência estratégica nas instituições pesquisadas esteja mais preparada e voltada para lidar com a questão das competências científicas relacionadas às doenças as quais os hospitais são orientados a focar do que as tecnológicas, evidenciando, com isso, a ineficiência na gestão de TI. Desse modo, seria lógico pressupor que a utilização de qualquer tecnologia, incluindo a Tecnologia da Informação, será subutilizada até que as organizações se conscientizarem da importância de desenvolver suas competências essenciais para a prestação de serviços de valor para a sociedade.

Considerando a complexidade, a problemática da área de saúde e as dificuldades encontradas na realização da presente pesquisa decorrentes da falta de participação das várias instituições convidadas, este trabalho apresenta limitações quanto à profundidade e representatividade do setor estudado. Assim, entende-se que muitas questões dessa área precisam ainda ser reformuladas e respondidas, e que futuros estudos que venham a contribuir para uma melhor identificação, compreensão e solução das causas da ineficiência da hospitalar terão espaços em pesquisas científicas.

\section{Notas}

1- Os autores expressam os seus agradecimentos aos revisores anônimos da revista pelas críticas construtivas e sugestões para a melhoria deste artigo, ao Conselho Editorial pelo profissionalismo e pela atenção aos pesquisadores, com fornecimento de informações durante o processo até a publicação do artigo, e à Profa. Angela Guiomar Nogueira pelas suas recomendações.

2- Este artigo representa um dos desdobramentos do Projeto GESITI/Saúde, projeto este coordenado pelo Professor Antonio José Balloni, pesquisador do CTI Renato Archer, de Campinas, SP e do qual participa um dos membros colaboradores deste projeto.

\section{Referências}

Aghaei, S., Nematbakhsh, M. A. \& Farsani, H. K. (2012). Evolution of the world wide web: From WEB 1.0 TO WEB 4.0. International journal Web \& Semantic Technology, 3(1), 1-10.

Albuquerque, J. P. (2009). Repensando processos de formalização em sistemas informatizados: analisando a co-evolução entre software e práticas organizacionais. Revista Eletrônica de Comunicação Informação e Inovação em Saúde, 3(2), 7-15.

Albuquerque, J. P, Prado, E. P. V., \& Machado, G. R. (2011). Ambivalent implications of health care information systems: a study in the Brazilian public health care system. Revista de Administração de Empresa [online], 51(1), 58-71.

Almeida, G. W., \& Melllo, R. C. (2004). Uso de novas tecnologias de informação por profissionais da área da saúde na Bahia. Revista de Administração Contemporânea, 8(3), 09-27.

Artmann, E. \& Rivera, F. J. U. (2003). A demarche stratégique (gestão estratégica hospitalar): um instrumento de coordenação da prática hospitalar baseada nos custos de oportunidade e solidariedade. Ciência \& Saúde Coletiva, 8(2), 478-499. 
Barbosa, J. G. P, Gonçalves, A. A., \& Simonetti, V. (2009). A proposed architecture for implementing a knowledge management system in the Brazilian National Cancer Institute. Brazilian Administration Review, $6(3), 247-262$.

Bardin, L. (2009). Análise de conteúdo (Ed. rev. e atual.). Lisboa: Edições 70.

Barney, J. B. H. (1991). Firms resources and sustained competitive advantage. Journal of Management, $17(1), 99-120$.

Barney, J. B. H. (1996). Gaining and sustaining competitive advantage. New York: Addison-Wesley Publishing Company.

Barney, J. B. H., \& Hesterly, W.S. (2007). Administração estratégica e vantagem competitiva (Ed. rev.). São Paulo: Pearson Prentice Hall.

Borba, V. R. (2011). Estratégia e ação: BSC no contexto das organizações de Saúde. Rio de Janeiro: Editora DOC.

Bryson, J. M., Ackermann, F., \& Eden, C. (2007). Putting the resource-based view of strategy and distinctive competencies to work in public organizations. Public Administration Review, 67(4), 702-717.

Capretz, M. A., Toledo, M. B. F., Fantinato, M., Garcia, D. Z. G., Wang, S., Allison, D., Nabuco, O., Rodrigues, M., Bonacin, R., Sasse, E. C., Gimenes, I., \& Cunha, A, B. (2009). Tecnologias de Internet em uma plataforma de colaboração para a pesquisa médica. Revista Eletrônica de Comunicação Informação e Inovação em Saúde, 3(4), 210-225.

Cavalcante, M. T. L., \& Vasconcellos, M. M. (2007). Tecnologia de informação para a educação na saúde: duas revisões e uma proposta. Ciência \& Saúde Coletiva, 12(3), 611-622.

Cheikhrouhou, N., Pouly, C. \& Choudhary, A. (2011). An empirical study on human and information technology aspects in collaborative enterprise networks. Journal of University of Computing and Science, $17(2), 203-223$.

Côrtes, P. L., \& Côrtes, E. G. (2011). Hospital information systems: a study of electronic patient records. Journal of Information Systems and Technical Management, 8(1), 134-154.

Doz, Y.,\& Hamel, G. (1998). Alliance advantage: the art of creating value through partnering. Boston, Massachusetts: Harvard Business School Press.

Duque, C. G. \& Lyra, M. R. (2010). The information architecture positioning in it governance. Brazilian journal of Information Science, 4(2), 38-43.

Eisenhardt,K. M., \& Martin, J. A. (2000). Dynamic capabilities: what are they? Strategic Management Journal, 21(10-11), 1105-1121.

Escrivão, J. A. (2007). Uso da informação na gestão de hospitais públicos. Ciência \& Saúde Coletiva, 12(3), 655-666.

Editora Finep (2011). Revista Finep: Inovação em pauta. Rio de Janeiro: 2(1): 54-57.

Freitas, H., \& Rech, I. (2003). Problemas e ações na adoção de novas tecnologias de informação. Revista de Administração Contemporânea, 7(1), 125-150.

Kaplan, R. S., \& Norton. D. P. (1992). The balanced scorecard: measures that drive performance. Harvard Business Review, 70(1), 71-79.

Levitt, T. (1985). A imaginação de marketing. (A. B. Simões, Trad.). São Paulo: Atlas.

Martins, G. A. (2006). Sobre confiabilidade e validade. Revista Brasileira de Gestão de Negócio, 8(20), 1-12.

Mintzberg, H., Ahlstrand, B., \& Lampel, J. (1998). Strategy Safari. New York: Free Press.

Newbert, S. L. (2007). Empirical research on the resource-based view of the firm: an assessment and suggestions for future research. Strategic Management journal, 28(2), 121-146.

Obrien, J. A, \& Maracas G. (2007). Administração de sistemas de informação: uma introdução (13ā. ed.). São Paulo: Mc GrawHill. 
Otto, D.T., Catanho, M., Degrave, W., \& Miranda, A. B. (2007). A plataforma PDTIS de bioinformática: da sequência à função. Revista Eletrônica de Comunicação Informação e Inovação em Saúde, 2(Sup.1), 288296.

Peteraf, M. A. (1993).The cornerstones of competitive advantage: resource-based view. Strategic Management journal, 14(3), 179-191.

Pinochet, L. H. C. (2007). O processo de formulação de uma política de segurança de informações segundo a percepção dos gestores: um estudo em instituições hospitalares. Tese de doutorado. Escola de Administração de Empresas da FGV, SP, Brasil.

Paiva Júnior, F. G.; Sousa Leão, A. L. M. D., \& Mello, S. C. B. (2011). Validade e Confiabilidade na Pesquisa Qualitativa em Administração. Revista de Ciências da Administração, 13(31), 190-209.

Prahalad, C.K., \& Hamel, G. (2005). Competindo pelo futuro (24a ed). Rio de Janeiro: Elsevier.

Queiroz, A. C. S., \& Moreira, D. A. (2007). Novas tecnologias e confiança nas organizações: um estudo de caso no contexto hospitalar. Revista de Negócios, 12(1), 42-55.

Resende, G. M. (2003). Um estudo do alinhamento estratégico da tecnologia da informação em hospitais. Tese de doutorado. Escola de Administração de Empresas da FGV, SP, Brasil.

Schreyögg, G..\& Kliesch-Ebertl, M. (2007). How dynamic can organizational capabilities be? Strategic Management Journal, 8(4), 913-933.

Sguario, V. M. G., \& Tomaél, M. I. (2011). Portal Corporativo Interno de um Hospital Universitário: um estímulo à aprendizagem organizacional. Encontros de Biblioteconomia: Revista Eletrônica de Biblioteconomia e Ciência da Informação, 16(32), 57-72.

Shengbin, H. B. Y. (2011). The impact of technology selection on innovation success and organizational performance. iBusiness, 3(1), 366-371.

Spil, T. A. M., \& Schuring, R. W. (2006). E-health systems diffusion and use: the innovation, the user and the use IT model. London: Idea Group Publishing.

Stoner, J. A. F., \& Freeman, R. E. (1985). Administração (5a ed.). (A. Calado, Trad.) Rio de Janeiro: LTC.

Teece, D. J. (2007). Explicating dynamic capabilities: the nature and micro foundations of (sustainable) firm performance. Strategic Management Journal, 28(13), 1319-1350.

Tellis, G. J, Prahbu, J. C., \& Chand, R. K. (2009). Radical innovation across nations: the preeminence of corporate culture. Journal of Marketing, 73(1), 3-23.

Vergara, S. C. (2000) Projetos e relatórios de pesquisa em Administração. São Paulo: Atlas.

Vergara, S. C. (2010). Métodos de pesquisa em Administração (4a ed.). São Paulo: Atlas.

Wernerfelt, B. (1984). A resource-based view of the firm. Strategic Management Journal, 5(1), 171-180.

Wu, I. L., \& Hsieh, P. J. (2011). Understanding hospital innovation enabled customer-perceived quality of structure, process, and outcome care. Total Quality Management \& Business Excellence, 22(2), 227-241.

Zarama-Vasquez, G., \& Vinck, D. (2008). Por que a informatização funciona? Estratégias de compensação dos atores no caso da prescrição médica. Revista Eletrônica de Comunicação Informação e Inovação em Saúde, 2(1), 19-28.

Zukerman, A. M. (2005). Health care strategic planning (2nd ed.). Ilinois: FACHE. 


\section{Apêndice I - Roteiro de Entrevistas}

1. Caracterização do Hospital

a. Nome:

b. Localização (cidade e bairro):

c. Vínculo/subordinação:

d. Número de funcionários (contratados + terceirizados):

e. Número de leitos:

f. Total de atendimentos/ano:

g. Total de internações/ano:

h. É referência local/regional em alguma área de saúde? Qual?

2. Realiza planejamento estratégico (PE)? Sim ( ); Não ()

a. Com que frequência: semestral ( ); anual (); Outro:

b. Como é feito? ___ _ _ Quem participa? Alta direção ( ); média gerência ( ); pessoal do nível operacional ()

c. Quais instrumentos de gestão são usados no PE? Matriz SWOT ( ); PDCA ( ); BSC ( ); KPI ( ); Análise de cenários ( ); Outros:

d. Quais são fontes de informação usadas no PE: internas ( ) i externas ( )

e. Quais são as bases usadas pelo hospital para formular a sua estratégia corporativa? 1) Intuição dos dirigentes ( ); 2) Análise da demanda ( ); 3) Análise de cenários ( ); 4) Outras fontes:

f. Quais são os mecanismos de avaliação de novas tecnologias, estratégias dos concorrentes e satisfação dos clientes?

g. Como é desdobrado o PE? 1) Em planos ( ); 2) Em programas; 3) Em ações; 4) Outros: desdobrado.

3. O hospital faz a prospecção de novas tecnologias? $\operatorname{Sim}($ ); Não ( );

a. Como é feita?

4. O hospital avalia a satisfação dos clientes? $\operatorname{Sim}($ ); Não ( )

a. Com que frequência: mensal () semestral; () anual ( ); Outro:

b. Como é feita?

5. O hospital avalia a satisfação dos funcionários? Sim ( ); Não ( )

a. Com que frequência: mensal () semestral; () anual ( ); Outro:

b. Como é feita?

6. O hospital possui algum programa (como 5S, por exemplo) ou sistema de gestão da qualidade próprio ou com base nas normas ISO implantado? Sim ( ); Não ()

a. Quais: e desde quando:

b. Tem acreditação? Sim ( ); Não ()

c. Costuma fazer benchmarking com interdepartamental? Sim ( ); Não ( )

i. Com que frequência?

ii. Que departamentos participam?

d. Costuma fazer benchmarking com outros hospitais? Sim ( ); Não ()

i. Com que frequência?

ii. Com quais hospitais?

e. Avalia o seu desempenho organizacional? Sim ( ); Não ( )

i. Com que frequência?

ii. O que é e como é avaliado?

iii. Quem avalia?

iv. Quais são os instrumentos de avaliação utilizados?

7. O hospital costuma investir em inovação tecnológica? Sim ( ); Não ( )

a. Qual foi o percentual dos seus recursos investido nos últimos 3 anos?

b. Quanto pretende investir no próximo ano?

c. Tem conhecimento sobre linhas de financiamento, crédito ou incentivos governamentais para a IT?

8. Quais são os seus principais entraves à inovação tecnologia: 1) baixa qualificação de pessoal de saúde ( ); 2) falta de capacitação do pessoal de $\mathrm{TI}($ ); 3) Não conhecimento da fontes de financiamento ( ); 4) Escassez de recursos financeiros; 5) Quadro de pessoal deficitário ( ); 6) Burocracia ( ); 7) Outros:

9. Quais são os recursos ou ferramentas de TI que possui? 1) Internet ( ); 2) Gerenciador de base de dados ( ); 3) Redes de comunicação ( ); 4) EAD ( ); 5) Gestão Hospitalar ( ); 6) Gestão de Estoque ( ); 6) Gestão Corporativa/ERP ( ); 7) Automação de escritório; 8) Sistema operacional ( ); 9) Servidores e outros periféricos ( ); 10) Comércio Eletrônico; 11) Outros:

10. Em quais recursos ou ferramentas de TI pretende investir no próximo ano e qual a prioridade (colocando entre parênteses a prioridade: 1 para a maior; 2 para a seguinte...)? Internet ( ); Gerenciador de base de dados ( ); Redes de comunicação ( ); EAD ( ); Gestão Hospitalar ( ); Gestão de Estoque (); Gestão Corporativa/ERP (); Automação de escritório; Sistema operacional ( ); Servidores e outros periféricos ( ); Comércio Eletrônico; Capacitação de pessoal ( ); Outros ():

11. O hospital possui parceria com: em com: 1) Centros de pesquisa ( ); 2) Universidades ( ); 3) Outras instituições de saúde ( ) - Quais:

12. Quais destas parcerias foram estabelecidas nos últimos 5 anos visando desenvolver atividades de inovação?

13. Quais foram os principais os fornecedores de recursos tecnológicos (P\&D), nos últimos 3 anos, considerando:

a. O seu porte?

b. A nacionalidade?

c. O percentual de investimentos realizados?

14. Como o hospital avalia o relacionamento com os potencias parceiros descritos no quadro abaixo, considerando os seguintes fatores para cada um deles?

a. O grau de importância: baixa (), média (), alta ()

b. Tipo de cooperação estabelecida: 1) Pesquisas de satisfação ( ); 2) Treinamento ( ); 3) Assistência Técnica ( ); 4) Ensaios para testes de produtos ( ); 4) P\&D ( ); 5) Outras atividades ( ). 
O planejamento estratégico e a visão baseada em recursos (RBV): uma avaliação da tecnologia da informação na gestão hospitalar

\begin{tabular}{|l|l|l|}
\hline \multicolumn{1}{|c|}{ Relações entre o hospital e: } & Importância & Tipo de Cooperação \\
\hline Clientes & & \\
\hline Fornecedores & & \\
\hline Outro hospital & & \\
\hline Empresas de consultoria & \\
\hline Universidades e institutos de pesquisa & & \\
\hline $\begin{array}{l}\text { Centros de capacitação profissional e } \\
\text { assistência técnica }\end{array}$ & & \\
\hline
\end{tabular}

15. Quais são os recursos/meios utilizados pelo hospital para o desenvolvimento de competências científicas (relacionadas à área de saúde)? 1) cursos de capacitação ( ); 2) palestras ( ); 3) Seminários/simpósios/congressos ( ); 4) Visitas técnicas ( ); 5) Outros:

16. Quais são os recursos/meios utilizados pelo hospital para o desenvolvimento de competências científicas (relacionadas à área de Tl e afins)? 1) cursos de capacitação ( ); 2) palestras ( ); 3) Seminários/simpósios/congressos ( ); 4) Visitas técnicas ( ); 5) Outros:

17. Que prioridade costuma ser atribuída pelo hospital em termos de frequência e percentual de investimento para: 1 ) 0 desenvolvimento de competências científicas? ( ); 2) O desenvolvimento de competências tecnológicas? ( ). 